\title{
Inflammation and ventricular-vascular coupling in hypertensive patients with metabolic syndrome
}

\author{
L. Zanoli a,1, A. Di Pino b,1, V. Terranova ${ }^{\text {b }}$, S. Di Marca ${ }^{\text {b }}$, M. Pisano ${ }^{\text {b }}$, R. Di Quattro ${ }^{\text {b }}$, \\ V. Ferrara ${ }^{\text {b }}$, R. Scicali ${ }^{\text {b }}$, A.M. Rabuazzo ${ }^{\text {b }}$, P. Fatuzzo ${ }^{\text {a }}$, P. Castellino ${ }^{\text {b }}$, S. Piro ${ }^{\text {b }}$, \\ F. Purrello ${ }^{b}$, L. Malatino ${ }^{\mathrm{b}, *}$ \\ ${ }^{a}$ Nephrology, Department of Clinical and Experimental Medicine, University of Catania, Catania, Italy \\ ${ }^{b}$ Internal Medicine, Department of Clinical and Experimental Medicine, University of Catania, Catania, Italy
}

Received 26 May 2018; received in revised form 29 July 2018; accepted 16 August 2018

Handling Editor: F. Galletti

Available online

\section{KEYWORDS \\ Arterial stiffness; \\ Chronic kidney \\ disease; \\ Inflammation; \\ Left ventricular mass \\ index; \\ Metabolic syndrome}

\begin{abstract}
Background and aims: Metabolic syndrome (MetS) is currently considered to raise the risk for type 2 diabetes and cardiovascular events. It has been suggested that part of this risk excess may be due to a cluster of additional factors associated with MetS. We aimed to investigate the role of inflammation on the ventricular-vascular coupling in patients with MetS. Methods and results: We enrolled a total of 227 hypertensive patients (106 with MetS and 121 without MetS) matched for age and gender. Aortic pulse wave velocity (aPWV), intima-media thickness (IMT) and high sensitivity C-reactive protein (CRP) increased according to the number of MetS components. Patients with MetS showed increased aPWV (11.5 $\pm 3.7 \mathrm{vs.} 10.3 \pm 2.5 \mathrm{~m} / \mathrm{s}$, $P=0.03)$ compared with controls. In a model adjusted for age, sex, heart rate and mean blood pressure, aPWV resulted increased in patients with CKD (beta $1.29 \mathrm{~m} / \mathrm{s}, 95 \% \mathrm{CI} 0.61-1.96 \mathrm{~m} / \mathrm{s}$, $P<0.001$ ) and MetS (beta $0.89 \mathrm{~m} / \mathrm{s}, 95 \% \mathrm{CI} 0.28-1.51 \mathrm{~m} / \mathrm{s}, P=0.005$ ). After additional adjustment for CRP and IMT, the slope of aPWV was respectively reduced by $16 \%$ and $62 \%$, suggesting that inflammation and intima-media thickening could contribute to aortic stiffening in patients with MetS. In these patients, aPWV was also associated with left-ventricular mass index (beta $0.79 \mathrm{~g} / \mathrm{m}^{2.7}, 95 \%$ CI $0.05-1.52 \mathrm{~g} / \mathrm{m}^{2.7}, P=0.05$ ).

Conclusion: MetS is characterized by an inflammation-dependent acceleration in cardiovascular ageing. This pattern of pathophysiological abnormalities may contribute to amplify the burden of cardiovascular risk in patients with MetS.

( 2018 The Italian Society of Diabetology, the Italian Society for the Study of Atherosclerosis, the Italian Society of Human Nutrition, and the Department of Clinical Medicine and Surgery, Federico II University. Published by Elsevier B.V. This is an open access article under the CC BY-NC-ND license (http://creativecommons.org/licenses/by-nc-nd/4.0/).
\end{abstract}

Acronyms: BP, blood pressure; CKD, chronic kidney disease; CRP, Creactive protein; EaI, effective arterial elastance; $E_{L V} I$, elastance of ventricular function; GFR, glomerular filtration rate; IMT, intimamedia thickness; LVMi, left ventricular mass index; MetS, metabolic syndrome; aPWV, aortic pulse wave velocity; TyG, triglyceride-glucose.

* Corresponding author. Nephrology, Department of Clinical and Experimental Medicine, Policlinico Universitario, University of Catania, Via Santa Sofia 78, 95123, Catania, Italy. Fax +3900390953782376.

E-mail address: dott.zanoli@gmail.com (L. Malatino).

${ }^{1}$ These authors have contributed equally.

\section{Introduction}

Metabolic syndrome (MetS) is associated with an increased risk of cardiovascular events, only in part attributable to the individual risk factors concurring to its definition [1]. The excess risk detected in patients with MetS could be ascribed to underlying factors other than its components since MetS predicted cardiovascular events 
over and above the predictive power of its individual components [1].

Increased blood pressure (BP) is an important component of MetS linked to subclinical organ damage, such as increased intima-media thickness (IMT), aortic pulse wave velocity (aPWV), left ventricular mass index (LVMi), and to chronic kidney disease (CKD). However, the mechanisms linking this association are not so far well defined in patients with MetS.

Chronic inflammation is a common feature of patients with MetS associated with increased LVMi [2]. We previously suggested that at least a part of the cardiovascular risk reported in patients with chronic inflammation could be mediated by an increase in aortic stiffness [3]. Aortic stiffness and reflected waves are increased in patients with chronic high-grade inflammation $[4,5]$ and associated with left ventricular function [6]. An increase in aortic stiffness has been also reported in patients with MetS $[7,8]$. Accordingly, an association between aortic stiffness and Creactive protein (CRP) was reported in patients with chronic high-grade inflammation [9] and in subjects with hypertension [10]. Interestingly, the inflammationdependent increase in arterial stiffness seems to be at least partly reversible and a potential target for therapy since anti-Tumor Necrosis Factor-alpha therapy was associated with a reduction in aPWV [5,11], whereas salicylates made the opposite [12]. Moreover, inflammation was also associated with left ventricular hypertrophy [13], a common feature of patients with MetS [14].

In this study, we hypothesized that chronic low-grade inflammation leads to an increased cardiovascular risk in patients with MetS due to an increase in aPWV and LVMi, two intermediate end points for cardiovascular events. Consequently, the aim of the present study was to investigate the role of inflammation in ventricular-vascular coupling in hypertensive patients with MetS.

\section{Methods}

\section{Study population}

This study was a multicentre, cross-sectional study conducted in the three Units of Internal Medicine (c/o Cannizzaro Hospital, Garibaldi Hospital and Academic Policlinic) of the Department of Clinical and Experimental Medicine of the University of Catania. We enrolled a total of 227 subjects with hypertension without previous cardiovascular events: 106 individuals with hypertension and MetS and 126 control subjects with hypertension, but without MetS, matched for age and sex.

Patients with coronary heart disease, congestive heart failure, severe mitral insufficiency, stroke, transient ischaemic attack, intermittent claudication and malignancies were excluded, as were smokers. Written informed consent was obtained from each patient included in the study. The study protocol conformed to the ethical guidelines of the 1975 Declaration of Helsinki and was previously approved by the institution's ethics committee on research on humans.

\section{Study design}

All of the participants were studied in a quiet room with a controlled temperature of $22 \pm 1{ }^{\circ} \mathrm{C}$ after $15 \mathrm{~min}$ of recumbent rest. In each subject, a non-invasive haemodynamic study was performed by an expert operator blinded to the clinical data and therapy. A second operator, blinded to the haemodynamic examination, collected the clinical data using a standardized questionnaire.

\section{Haemodynamic data}

The non-invasive study of haemodynamic variables was performed as previously reported [15] in a centralized vascular laboratory. Briefly, brachial BP measurements were performed using an oscillometric device (Dinamap ProCare 100; GE Healthcare, Milwaukee, WI, USA). aPWV was measured by a SphygmoCor device (SphygmoCor system ${ }^{\circledR}$, AtCor Medical, Sydney, Australia) using the footto-foot velocity method, the intersecting tangent algorithm and the direct distance between the measurement sites [16]: $\operatorname{aPWV}(\mathrm{m} / \mathrm{s})=0.8 \cdot[$ direct distance $(\mathrm{m}) / \Delta \mathrm{t}]$. The radial pulse wave profile was recorded by applanation tonometry (SphygmoCor system ${ }^{\circledR}$, AtCor Medical, Sydney, Australia) after recalibration with brachial systolic and diastolic BP in the contralateral arm. The central pulse wave profile was constructed using the generalized transfer function, from which the central BPs and endsystolic pressure were derived as previously described and validated [17].

Systemic vascular resistance was calculated as the central mean BP divided by cardiac output; effective arterial elastance (EaI) was calculated as end-systolic pressure/stroke volume indexed for body surface area; elastance of ventricular function $\left(\mathrm{E}_{\mathrm{LV}} \mathrm{I}\right)$ was calculated as end-systolic pressure/end-systolic volume indexed for body surface area; and arterial-ventricular coupling index (AVCI) was calculated as $\mathrm{EaI} / \mathrm{E}_{\mathrm{LV}} \mathrm{I}$.

IMT was measured $2 \mathrm{~cm}$ below the right carotid bulb using a high-precision echotracking device (MyLab 30 Gold, Esaote, Maastricht, Netherlands), paired with a high resolution linear array transducer $(11 \mathrm{MHz})$.

\section{Echocardiography}

A trans-thoracic 2D-echocardiography was performed. Echocardiographic data were available in all hypertensive patients with MetS, but only in a minority of hypertensive patients without MetS. A MyLab 30 Gold (Esaote, Maastricht, Netherlands) was used for this purpose, with a 2.0-3.5 $\mathrm{MHz}$ transducer (see supplementary data).

\section{Definitions of risk factors}

Diagnosis of MetS was defined according to the revised NCEP ATP III by the presence of at least 3 of the following factors: 
- High BP: systolic/diastolic BP $\geq 130 / 85 \mathrm{~mm} \mathrm{Hg}$ or treatment for hypertension;

- Abdominal obesity: waist circumference $>102 \mathrm{~cm}$ for men and $>88 \mathrm{~cm}$ for women;

- High triglycerides: $\geq 1.7 \mathrm{mmol} / \mathrm{l}(150 \mathrm{mg} / \mathrm{dl})$ or specific treatment for this lipid abnormality;

- Low HDL cholesterol: $<1.03 \mathrm{mmol} / \mathrm{l}(40 \mathrm{mg} / \mathrm{dl})$ in men and $<1.29 \mathrm{mmol} / \mathrm{l}(50 \mathrm{mg} / \mathrm{dl})$ in women or specific treatment for this lipid abnormality; and

- High fasting plasma glucose: $\geq 5.6 \mathrm{mmol} / \mathrm{l}(100 \mathrm{mg} / \mathrm{dl})$ or previously diagnosed type 2 diabetes.

\section{Biological variables}

Standard laboratory data were measured 1-7 days before the haemodynamic study in our centralized laboratory. CKD was defined as glomerular filtration rate (calculated using the CKD-EPI creatinine equation [18]) $<60 \mathrm{ml} / \mathrm{min} /$ $1.73 \mathrm{~m}^{2}$. The triglyceride-glucose (TyG) index, a measure linked to insulin resistance in the muscle, was calculated as $\ln$ [fasting triglycerides -fasting glucose/2]; the insulin resistance index of homeostasis model assessment (HOMA-IR), a marker of insulin resistance in the liver, was also calculated. High sensitivity CRP was measured using a commercially available kit (Behring, Scoppito, L'aquila, Italy; normal values $<1 \mathrm{mg} / \mathrm{dl}$ ).

\section{Statistical analysis}

We determined the sample size adequate to demonstrate that patients with MetS have a higher aPWV than control subjects (see Supplementary data). Continuous variables are presented as means (standard deviations), and categorical variables are presented as percentages. A positively skewed continuous variable (CRP) was log-transformed. Clinical and haemodynamic variables were compared using the Kruskal-Wallis test for continuous variables and the chi-square tests for categorical variables in univariate analyses. Outlier-robust multivariate linear regression analyses were used to evaluate the factors associated with aPWV and LVMi. A two-tailed $P$-value $<0.05$ was considered statistically significant. Statistical analyses were performed using NCSS 2007 and PASS 11 software (Gerry Hintze, Kaysville, UT, USA).

\section{Results}

\section{Clinical characteristics of patients with MetS}

The main clinical data are presented in Supplementary Table 1 . The matching process worked well since age and sex were comparable between the patients with hypertension without MetS and those with MetS, as were mean BP and GFR. Subjects with MetS had higher CRP (0.31 \pm 0.44 vs. $0.04 \pm 0.48 \log (\mathrm{mg} / \mathrm{l}), P<0.001)$, TyG Index $(9.08 \pm 0.64$ vs. $8.39 \pm 0.43)$, IMT $(920 \pm 199$ vs. $719 \pm 123 \mu \mathrm{m}, P<0.001)$ and $\operatorname{aPWV}(11.5 \pm 3.7$ vs. $10.3 \pm 2.5 \mathrm{~m} / \mathrm{s}, P=0.03$ ) compared to controls. Accordingly, CRP, TyG index, IMT and aPWV increased according to the number of MetS components (Supplementary Fig. 12). There was no influence of gender on aPWV values.

\section{Inflammation and ventricular-vascular coupling in patients with MetS}

The main haemodynamic data of patients with MetS are reported in Table 1. CRP was positively associated with IMT (Fig. 1, Panel A) and with measures of insulin resistance (TyG index, Supplementary Fig. 2, Panel B), arterial stiffness (aPWV and Eal, Fig. 1, Panel B and Supplementary Fig. 3, Panel A), left ventricular stiffness ( $\mathrm{E}_{\mathrm{LV}} \mathrm{I}$, Supplementary Fig. 3, Panel B) and mass (LVMi, Supplementary Fig. 3, Panel C).

The increase in aPWV in subjects with MetS was confirmed in a model adjusted for age, sex, heart rate and mean BP (beta $1.29 \mathrm{~m} / \mathrm{s}, 95 \%$ CI 0.61-1.96; $P=0.005$;

Table 1 Ventricular-vascular coupling and insulin resistance in patients with metabolic syndrome with and without chronic kidney disease.

\begin{tabular}{|c|c|c|c|c|}
\hline \multirow[t]{2}{*}{ Variables } & \multirow[t]{2}{*}{ All MetS patients } & \multirow{2}{*}{$\frac{\text { Without CKD }}{\mathrm{A}}$} & \multirow{2}{*}{$\frac{\text { With CKD }}{\text { B }}$} & \multirow{2}{*}{$\frac{P \text {-value }}{A-B}$} \\
\hline & & & & \\
\hline Patients, $\mathrm{n}$ & 106 & 81 & 25 & \\
\hline TyG index & $9.1(0.6)$ & $9.1(0.7)$ & $9.1(0.5)$ & 0.96 \\
\hline HOMA-IR & $8.6(9.0)$ & $7.8(9.0)$ & $11.0(8.8)$ & 0.14 \\
\hline Heart rate, $\mathrm{b} / \mathrm{min}$ & $70(10)$ & $71(10)$ & $65(10)$ & 0.02 \\
\hline Central mean BP, mm Hg & $98(13)$ & $99(13)$ & $95(15)$ & 0.23 \\
\hline Intima-media thickness, $\mu \mathrm{m}$ & $912(179)$ & $917(184)$ & $895(166)$ & 0.66 \\
\hline Aortic PWV, m/s & $11.5(3.7)$ & $11.1(3.8)$ & $12.7(3.0)$ & 0.02 \\
\hline $\mathrm{EaI}, \mathrm{mm} \mathrm{Hg} / \mathrm{ml} / \mathrm{m}^{2}$ & $0.88(0.26)$ & $0.85(0.26)$ & $0.98(0.25)$ & 0.02 \\
\hline $\mathrm{E}_{\mathrm{LV}} \mathrm{I}, \mathrm{mm} \mathrm{Hg} / \mathrm{ml} / \mathrm{m}^{2}$ & $2.92(1.40)$ & $2.98(1.51)$ & $2.69(0.95)$ & 0.64 \\
\hline AVCI & $0.35(0.16)$ & $0.34(0.17)$ & $0.39(0.13)$ & 0.04 \\
\hline SVR, dyne $\cdot \mathrm{s} \cdot \mathrm{cm}^{-5}$ & $1710(412)$ & 1695 (399) & $1757(459)$ & 0.54 \\
\hline Relative wall thickness & $0.46(0.07)$ & $0.47(0.07)$ & $0.44(0.07)$ & 0.10 \\
\hline LVMi, $\mathrm{g} / \mathrm{m}^{2.7}$ & $46.2(14.8)$ & $44.0(14.1)$ & $53.4(15)$ & 0.002 \\
\hline
\end{tabular}

${ }^{*}$ Available in 58 participants without CKD and 25 participants with CKD. AIx, Augmentation index; AVCI, arterial-ventricular coupling index; BP, blood pressure; EaI, effective arterial elastance normalized for body surface area; $E_{\mathrm{LV}}$, elastance of ventricular function normalized for body surface area; LVMi, left-ventricular mass indexed for height ${ }^{2.7}$; PWV, pulse wave velocity; SVR, systemic vascular resistance; TyG, triglycerideglucose. Significant $P$-values are in bold. 

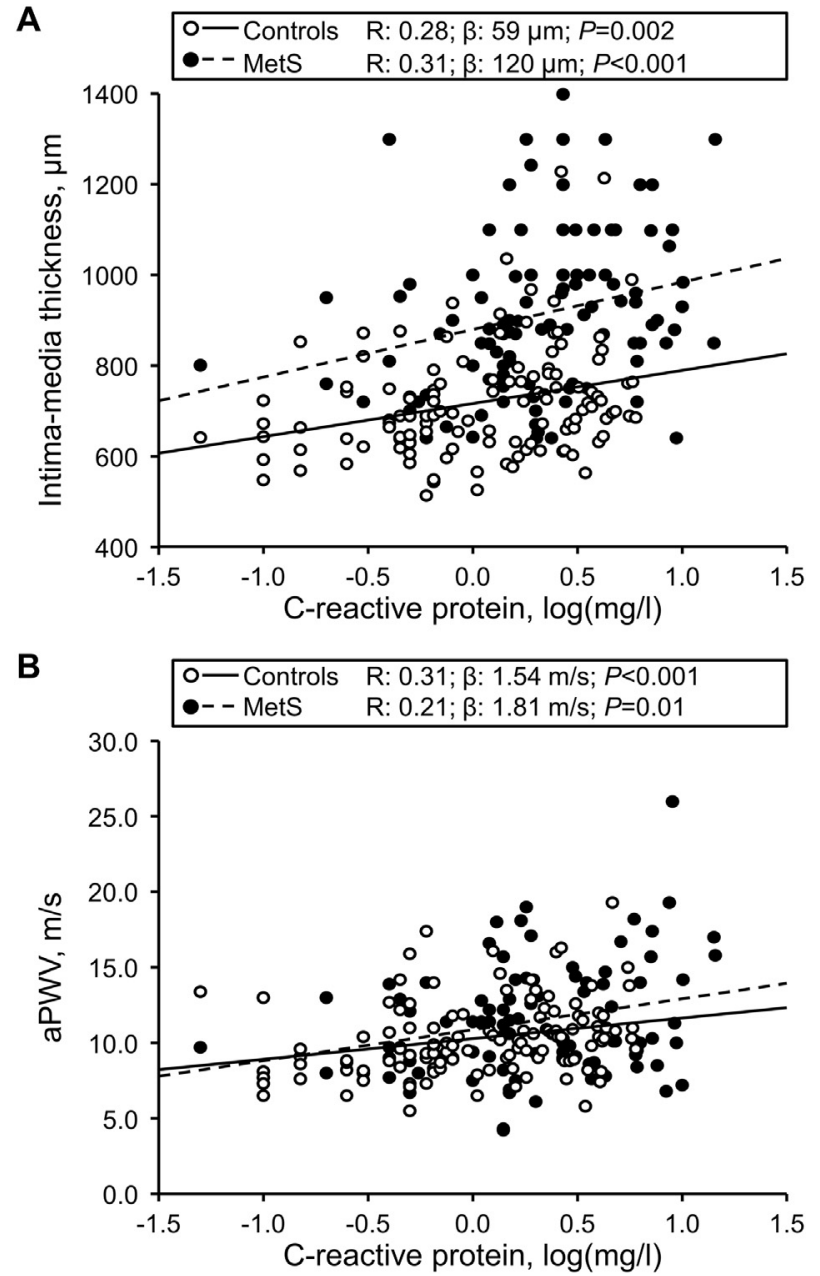

Figure 1 Association between C-reactive protein and intima-media thickness (Panel A) or aortic pulse wave velocity (aPWV) (Panel B). MetS, metabolic syndrome.

Table 2, Model 1). To evaluate the effect of inflammation on aPWV, we performed a stepwise analysis including in the model CRP (Table 2, Model 2) and then IMT (Table 2, Model 3); the increase in aPWV in subjects with MetS was respectively reduced by $16 \%$ (beta $0.75 \mathrm{~m} / \mathrm{s}, 95 \% \mathrm{CI}$ $0.12-1.37 \mathrm{~m} / \mathrm{s}, P=0.02$ ) and $62 \%$ (beta $0.34 \mathrm{~m} / \mathrm{s}, 95 \% \mathrm{CI}$ $-0.35-1.02 \mathrm{~m} / \mathrm{s}, P=0.33)$. The association between the components of MetS and aPWV is reported in Supplementary Table 2.

In patients with MetS, aPWV was directly associated with LVMi and negatively associated with RWT (Fig. 2, Panel B-C). The association between aPWV and LVMi was confirmed in a multivariate model adjusted for age, sex, heart rate and mean BP (beta $0.79 \mathrm{~g} / \mathrm{m} 2.7,95 \% \mathrm{CI}$ 0.05-1.52 g/m2.7, $P=0.04$; Table 2, Model 4), as well as the association between aPWV and RWT (beta -0.0042 , $95 \% \mathrm{CI}-0.0078$ to $-0.0007, P=0.02$ ). After additional adjustment for CRP (Table 2, Model 5), the association between LVMi and aPWV was lost, whereas CKD emerged as the only significant predictor of LVMi, therefore emphasizing that the weight of covariates is captured by CKD, a phenotype largely recognized to be linked with microinflammation.
Moreover, we tested whether ventricular-vascular coupling was altered in patients with MetS and CKD (Table 1). For a comparable mean BP and IMT, MetS patients with CKD had higher aPWV and LVMi than those without CKD, as well as an increased AVCI, since EaI was increased in the former group, whereas $\mathrm{E}_{\mathrm{LV}} \mathrm{I}$ was comparable between the patients with and without CKD.

\section{Discussion}

This study shows that early vascular ageing and an increased left ventricular stiffness and mass were both evident in patients with MetS and associated with inflammation (Fig. 3, Panel A-C).

According to the Literature, we observed that CRP was associated with IMT and aPWV in patients with MetS. In this respect, several mechanisms can lead to inflammation-dependent aortic stiffening [19]. Inflammation can result in functional (i.e., endothelial dysfunction) and structural arterial stiffening (i.e., increased activity of metalloproteinases, with the consequent production of elastases, gelatinases and collagenases, hypertrophy and reduced apoptosis of smooth muscle cells, and vascular calcification) in patients with MetS. Moreover, it is of interest that intima-media thickening can lead, by itself, to structural arterial stiffening. Consequently, the increased IMT, reported in patients with MetS in this and previous studies, could have a role on the stiffening of the arterial wall in these subjects. Of note, this relationship between an increase in carotid IMT and stiffening of the arterial wall, shown in the present study, was not simultaneously considered in previous studies performed in patients with MetS. Interestingly, we reported that the beta of aPWV in subjects with MetS was reduced by $16 \%$ after adjustment for CRP (Table 2, Model 1-2) and by $62 \%$ in a model that also included IMT (Table 2, Model 1-3). Moreover, in the latter model, the association between CRP and aPWV was lost when IMT is included in the model. Therefore, taken together, our data suggest that the increase in aPWV in patients with MetS could be dependent on inflammation and associated with the increase in IMT. In this regard, the association of aPWV with fasting hyperglycaemia (Supplementary Table 2) and TyG index (Supplementary Fig. 2, Panel C) confirmed that hyperinsulinaemia and hyperglycaemia would be important players in the development of vascular inflammation even in patients with MetS.

There has been evidence that MetS is associated with CKD [20], a complex phenotype in which increased aortic stiffness is commonly reported, and that CKD can lead to cardiovascular impairment [21]. In the present study, we reported that, compared to MetS patients without CKD, aPWV was increased in those with CKD, whereas IMT was comparable between groups (Table 1). These data are in keeping with similar findings in CKD patients without MetS [22], and suggest that aortic stiffening could proceed even without thickening of the arterial wall in hypertensive patients with MetS and CKD. Moreover, despite aPWV was increased in patients with CKD (Table 1), the slope of 
Table 2 Stepwise multivariate analysis. Clinical and biochemical determinants of aortic pulse wave velocity and left-ventricular mass index.

\begin{tabular}{|c|c|c|c|c|}
\hline & Units & Beta & $\mathrm{R}^{2}$ & $P$-value \\
\hline \multicolumn{5}{|c|}{ Determinants of aortic pulse wave velocity $(\mathrm{m} / \mathrm{s})$} \\
\hline \multicolumn{5}{|l|}{ Model 1} \\
\hline CKD & Yes & $1.29(0.61-1.96)$ & 0.05 & $<\mathbf{0 . 0 0 1}$ \\
\hline MetS & Yes & $0.89(0.28-1.51)$ & 0.02 & 0.005 \\
\hline \multicolumn{5}{|l|}{ Model 2} \\
\hline CKD & Yes & $1.25(0.59-1.92)$ & 0.04 & $<\mathbf{0 . 0 0 1}$ \\
\hline MetS & Yes & $0.75(0.12-1.37)$ & 0.02 & 0.02 \\
\hline C-reactive protein & $\log (\mathrm{mg} / \mathrm{l})$ & $0.69(0.02-1.36)$ & 0.01 & 0.04 \\
\hline \multicolumn{5}{|l|}{ Model 3} \\
\hline CKD & Yes & $1.36(0.71-2.02)$ & 0.05 & $<\mathbf{0 . 0 0 1}$ \\
\hline MetS & Yes & $0.34(-0.35$ to 1.02$)$ & 0.00 & 0.33 \\
\hline C-reactive protein, log (mg/l) & $\log (\mathrm{mg} / \mathrm{l})$ & $0.52(-0.16$ to 1.20$)$ & 0.01 & 0.13 \\
\hline Intima-media thickness & $100 \mu \mathrm{m}$ & $0.28(0.07-0.49)$ & 0.02 & 0.01 \\
\hline \multicolumn{5}{|c|}{ Determinants of left ventricular mass index $\left(\mathrm{g} / \mathrm{m}^{2.7}\right)$ in patients with MetS } \\
\hline \multicolumn{5}{|l|}{ Model 4} \\
\hline CKD & Yes & $6.89(0.90-12.88)$ & 0.04 & 0.02 \\
\hline $\mathrm{aPWV}, \mathrm{m} / \mathrm{s}$ & $\mathrm{m} / \mathrm{s}$ & $0.79(0.05-1.52)$ & 0.04 & 0.04 \\
\hline \multicolumn{5}{|l|}{ Model 5} \\
\hline CKD & Yes & $6.97(0.94-13.00)$ & 0.04 & 0.02 \\
\hline $\mathrm{aPWV}, \mathrm{m} / \mathrm{s}$ & $\mathrm{m} / \mathrm{s}$ & $0.67(-0.08$ to 1.42$)$ & 0.03 & 0.08 \\
\hline C-reactive protein, $\log (\mathrm{mg} / \mathrm{l})$ & $\log (\mathrm{mg} / \mathrm{l})$ & $3.84(-2.08$ to 9.77$)$ & 0.01 & 0.20 \\
\hline
\end{tabular}

Outlier-robust multivariate linear regressions show the variables that were significantly associated with the aortic pulse wave velocity and left ventricular mass index. aPWV indicates aortic pulse wave velocity; CKD, chronic kidney disease; MetS, metabolic syndrome. Adjusted for age, sex, heart rate and mean blood pressure. Significant $P$-values are in bold.

CKD for aPWV was not modified by CRP (Table 2, Model $1-2)$, suggesting that the role of CKD in arterial stiffening in patients with MetS could be additive to that of inflammation.

It was previously reported that left ventricular remodelling was associated with the inflammatory status in patients with and without MetS [2]. Here we also reported that ventricular-vascular coupling, as assessed by EaI and $E_{\mathrm{LV}} \mathrm{I}$, could be influenced by inflammatory status and CKD in hypertensive patients with MetS. It is well recognized that EaI is an index of the functional properties of the whole arterial system [23], while $E_{\mathrm{LV}} \mathrm{I}$ is an index of ventricular chamber contractility and a useful parameter to distinguish a normal from a failing ventricle relatively insensitive to load conditions [24]. Both EaI and $\mathrm{E}_{\mathrm{LV}} \mathrm{I}$, together with LVMi, were all associated with CRP in patients with MetS. Moreover, compared with patients with MetS without CKD, those with CKD had increased EaI and comparable $\mathrm{E}_{\mathrm{LV}} \mathrm{I}$. Consequently, $\mathrm{AVCI}$ was also increased in hypertensive patients with MetS and CKD, suggesting that the alterations of the vascular function exceed those of ventricular function in this specific clinical setting.

It is of interest that, although CKD, even in the early stages, is somehow associated with insulin resistance, our results did not show an additional effect of CKD on insulin resistance in presence of MetS, looking as if the impact of MetS would overwhelm that of CKD. However, the small number of patients with CKD does not allow any conclusion regarding this issue, although HOMA-IR shows a trend to increase in patients with CKD (Table 1). Moreover, it should be emphasized, in this respect, that HOMA-IR utilizes fasting levels of insulin, that are higher in CKD patients due to a reduced renal clearance of this peptide. Consequently, although HOMA-IR is commonly used to assess insulin sensitivity in clinical practice, hyperinsulinemic euglycemic clamp can better than HOMA-IR reflect the degree of insulin resistance in CKD patients [25].

It is of interest that in hypertensive patients with MetS, increased aPWV was associated positively with LVMi and negatively with RWT (Fig. 2, Panel B-C). It is known that increased arterial stiffness leads to an early return of the reflected waves and augmentation of late systolic pressure, increased workload of the left ventricle and left ventricular hypertrophy [6]. Whether arterial stiffening is associated with eccentric or concentric left ventricular hypertrophy in patients with MetS is not clear. Our finding is suggestive of the development of eccentric left ventricular remodelling in hypertensive patients with MetS showing an increased aortic stiffness. Moreover, the trend for a reduction in ejection fraction reported in MetS patients with increased aPWV $(P=0.11)$ runs in parallel with the concept that these patients may develop dilated cardiomyopathy with left ventricular dysfunction. The association between CRP and $\mathrm{E}_{\mathrm{LV}} \mathrm{I}$ confirmed the role of systemic inflammation in this process [26]. However, the lack of correlation between LVMi and $\mathrm{E}_{\mathrm{LV}} \mathrm{I}(P=0.35)$ may suggest that hypertrophy and stiffening of the left ventricle proceed independently in hypertensive patients with MetS.

Finally, we reported no influence of gender on aPWV values. In the Literature, the role of gender on aPWV is still debated since some authors described a gender-related difference in aPWV [27] whereas others reported the opposite [28]. Further studies are needed to clarify this issue. 
A

๑- - MetS R: $0.29 ; \beta: 0.5 \mathrm{~m} / \mathrm{s}$ for $100 \mu \mathrm{m} ; P=0.001$

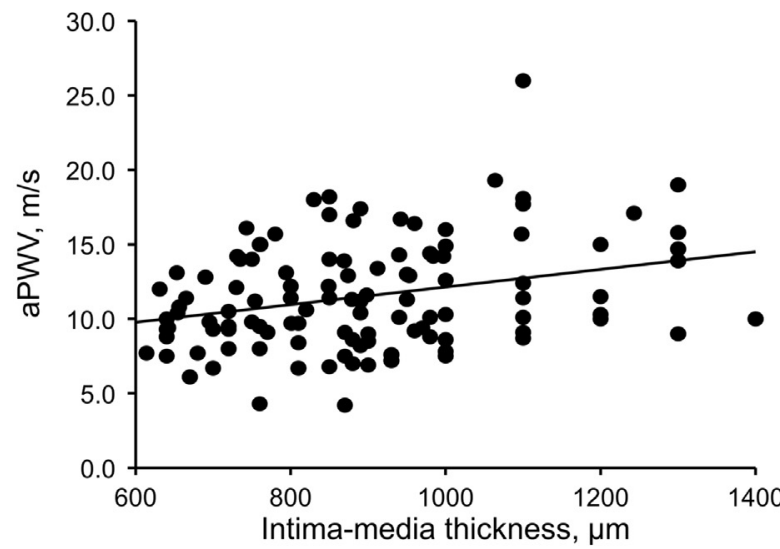

B

$\bullet--$ MetS R: $0.26 ; \beta: 1.1 \mathrm{~g} / \mathrm{m}^{2.7} ; P=0.001$

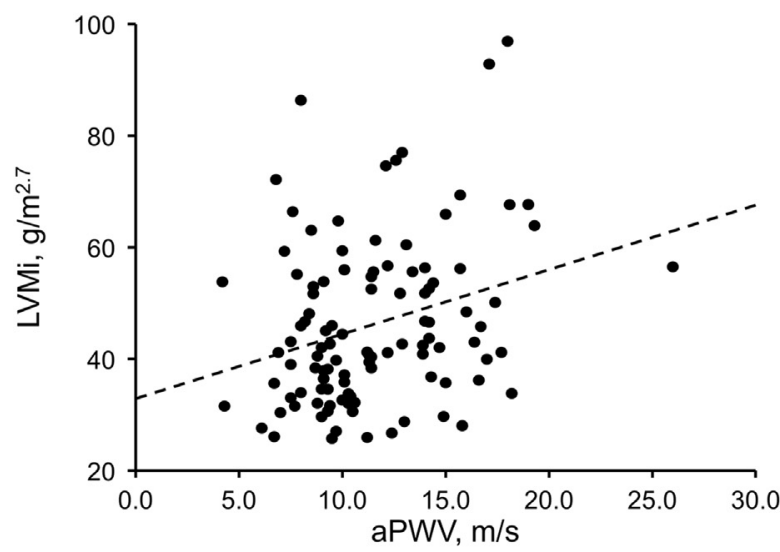

C
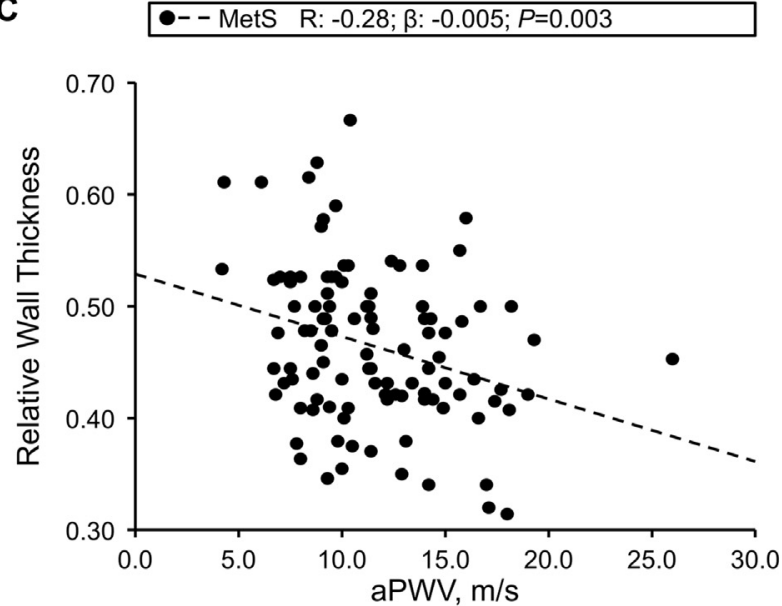

Figure 2 Association between aortic pulse wave velocity (aPWV) and intima-media thickness (Panel A), left-ventricular mass index (LVMi) (Panel B) or relative wall thickness (Panel C). MetS, metabolic syndrome.

\section{Methodological issues}

The present study has several strengths. First, it was the first evaluating the role of inflammation and CKD in ventricular-vascular coupling in hypertensive patients with MetS. Second, we enrolled only patients with
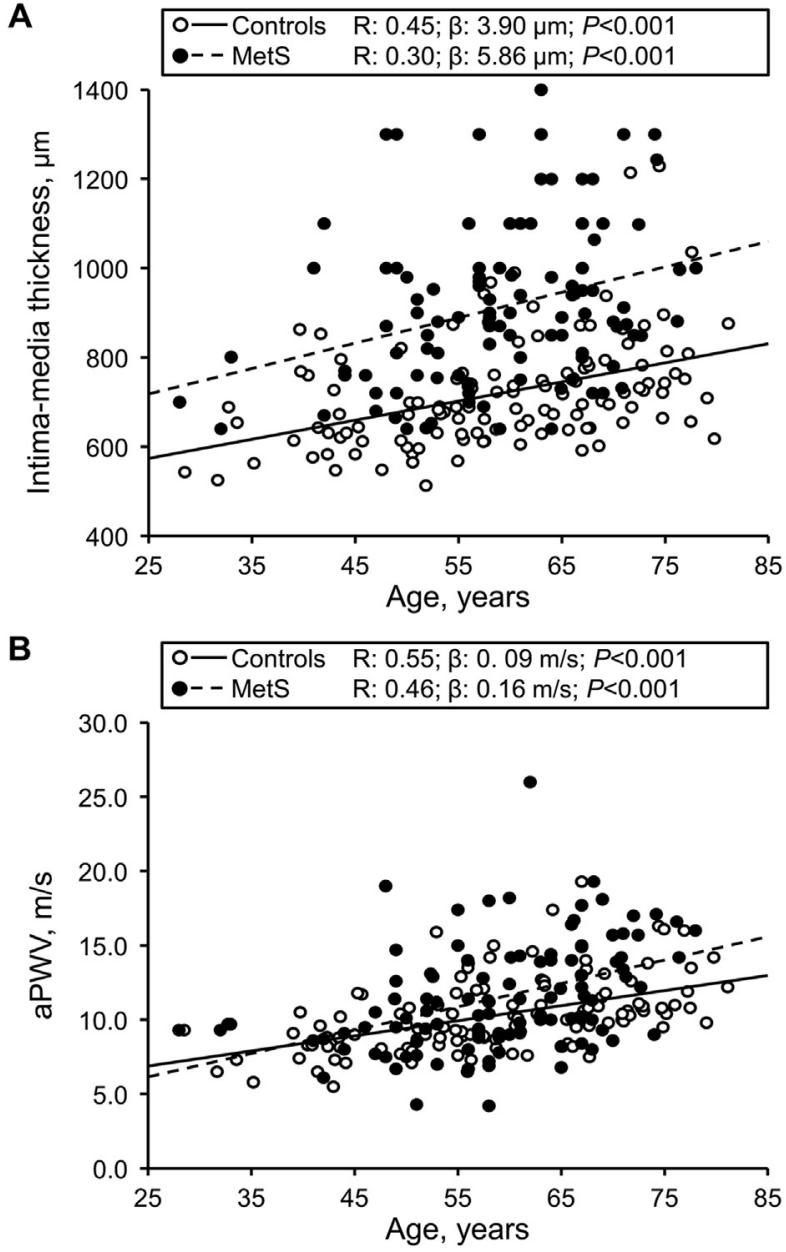

C

\section{Metabolic syndrome}

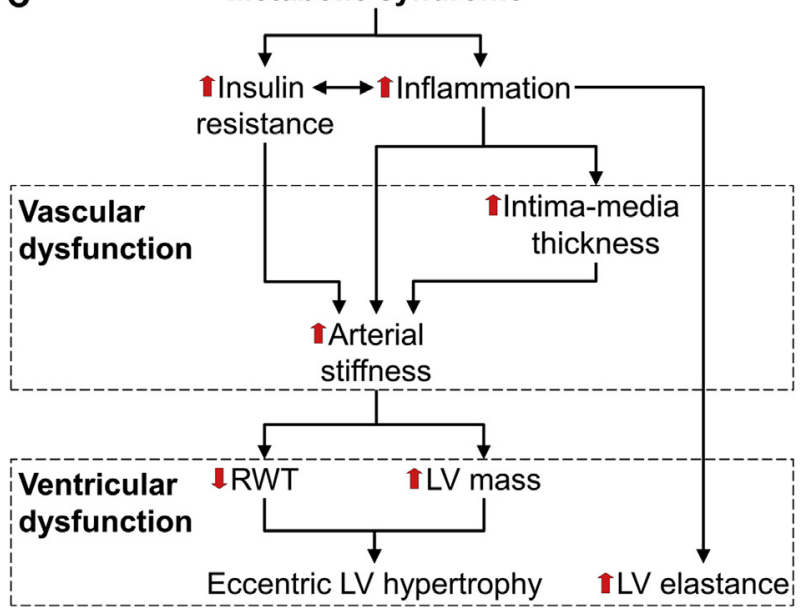

Figure 3 Panel A-B: Early vascular aging in patients with metabolic syndrome (MetS); Panel C: Inflammation and ventricular-vascular coupling in patients with metabolic syndrome. aPWV, aortic pulse wave velocity; LV, left ventricular; RWT, relative wall thickness.

hypertension, both in the control group and in the MetS group. This study design allowed us to evaluate the additive effects of the components of MetS on ventricularvascular coupling in patients with hypertension. Finally, we used a well-validated method to calculate end-systolic pressure [17]. 
This study has also some limitations. First, it was a cross-sectional study. Therefore, all associations should be confirmed in a longitudinal study. Second, we failed to perform a comparison of left ventricular measurements between hypertensive patients with and without MetS, because echocardiographic data were available only in a small number of hypertensive patients without MetS. However, it is known that the prevalence of leftventricular hypertrophy is increased in hypertensive patients with MetS as compared with those without MetS [14]. Third, we have no data on physical activity and Vitamin D. It is known that physical activity can potentially affect both inflammation and arterial stiffening, whereas Vitamin D may play a pivotal role in modulating insulin resistance [29] and is inversely associated with increased arterial stiffness [30]. In particular, low Vitamin D levels are associated with vascular inflammation, endothelial dysfunction, formation of foam cells, and proliferation of smooth muscle cells. Further studies are needed to clarify the role of physical activity and Vitamin D on ventricularvascular coupling in patients with MetS.

\section{Conclusions}

In hypertensive patients with MetS, cardiovascular ageing was accelerated and dependent on inflammation. This outcome could suggest that, in hypertensive patients with MetS, long-term monitoring of inflammation along with aortic stiffness, an established surrogate measure of cardiovascular risk, may provide clinicians with both additional information on the severity of cardiovascular dysfunction, and a potential target of therapy.

\section{Conflicts of interest}

None.

\section{Contributorship statement}

All authors meet the ICMJE Recommendations for authorship credit.

ZL and ML have contributed substantially to the conception and design of the study, analysis and interpretation of data and drafting the paper; FP, PF, PS, RAM and $\mathrm{CP}$ to the design of the study, interpretation of data, revising the work critically for important intellectual content; DPA, TV, DMS, PM, DQR, FV and SR to the acquisition of data and revising the work critically for important intellectual content. All Authors have read and approved the manuscript and agree to be accountable for all aspects of the work in ensuring that questions related to the accuracy or integrity of any part of the work are appropriately investigated and resolved.

\section{Acknowledgements}

This study was funded by 2016/2018 Department Research Plan of University of Catania, Department of Clinical and
Experimental Medicine (Project \#A) and by the Ministry of Health, Italy (GR-2011-02349066).

\section{Appendix A. Supplementary data}

Supplementary data related to this article can be found at https://doi.org/10.1016/j.numecd.2018.08.003.

\section{References}

[1] Scuteri A, Najjar SS, Morrell CH, Lakatta EG. The metabolic syndrome in older individuals: prevalence and prediction of cardiovascular events: the Cardiovascular Health Study. Diabetes Care 2005;28:882-7.

[2] Bo S, Mandrile C, Milanesio N, Pagani A, Gentile L, Gambino R, et al. Is left ventricular hypertrophy a low-level inflammatory state? A population-based cohort study. Nutr Metab Cardiovasc Dis 2012;22(8):668-76.

[3] Zanoli L, Inserra G, Castellino P. Increased cardiovascular risk in subjects with a low prevalence of classic cardiovascular risk factors: the inflammatory bowel disease paradox. Trends Cardiovasc Med 2015;25(8):705-6. https://doi.org/10.1016/j.tcm.2015.04.001.

[4] Zanoli L, Rastelli S, Granata A, Inserra G, Empana JP, Boutouyrie P, et al. Arterial stiffness in inflammatory bowel disease: a systematic review and meta-analysis. J Hypertens 2016;34(5):822-9. https://doi.org/10.1097/HJH.0000000000000867.

[5] Zanoli L, Granata A, Lentini P, Gaudio A, Castellino P. Augmentation index is increased in patients with inflammatory bowel disease, a meta-analysis. Eur J Intern Med 2017;39:e31-2. https: //doi.org/10.1016/j.ejim.2016.12.012.

[6] Aslan AN, Sarı C, Özer Sarı S, Tayfur Yürekli Ö, Baştuğ S, Sivri S, et al. Association between aortic stiffness and left ventricular function in inflammatory bowel disease. Cardiol J 2016;23(2): 202-10. https://doi.org/10.5603/CJ.a2016.0008.

[7] Zanoli L, Empana JP, Estrugo N, Escriou G, Ketthab H, Pruny JF, et al. The neural baroreflex pathway in subjects with metabolic syndrome: a sub-study of the paris prospective study III. Medicine (Baltimore) 2016;95(2):e2472. https://doi.org/10.1097/MD.0000000000002472.

[8] Topouchian J, Labat C, Gautier S, Bäck M, Achimastos A, Blacher J, et al. Effects of metabolic syndrome on arterial function in different age groups: the Advanced Approach to Arterial Stiffness study. J Hypertens 2018;36(4):824-33. https://doi.org/10.1097/ HJH.0000000000001631.

[9] Zanoli L, Boutouyrie P, Fatuzzo P, Granata A, Lentini P, Oztürk K, et al. Inflammation and aortic stiffness: an individual participant data meta-analysis in patients with inflammatory bowel disease. J Am Heart Assoc 2017;6(10). https://doi.org/10.1161/JAHA.117.007003.

[10] Pietri P, Vyssoulis G, Vlachopoulos C, Zervoudaki A, Gialernios T, Aznaouridis K, et al. Relationship between low-grade inflammation and arterial stiffness in patients with essential hypertension. J Hypertens 2006;24(11):2231-8.

[11] Vlachopoulos C, Gravos A, Georgiopoulos G, Terentes-Printzios D, Ioakeimidis N, Vassilopoulos D, et al. The effect of TNF-a antagonists on aortic stiffness and wave reflections: a meta-analysis. Clin Rheumatol 2018;37(2):515-26. https://doi.org/10.1007/s10067017-3657-y.

[12] Zanoli L, Boutouyrie P, Lentini P, Rastelli S, Castellino P. Maintenance therapy with salicylates is associated with aortic stiffening in patients with inflammatory bowel disease. J Hypertens 2017 Apr;35(4):898-9. //doi.org/10.1097/HJH.0000000000001235.

[13] Conen D, Zeller A, Pfisterer M, Martina B. Usefulness of B-type natriuretic peptide and $\mathrm{C}$-reactive protein in predicting the presence or absence of left ventricular hypertrophy in patients with systemic hypertension. Am J Cardiol 2006;97(2):249-52.

[14] Cuspidi C, Sala C, Lonati L, Negri F, Rescaldani M, Re A, et al. Metabolic syndrome, left ventricular hypertrophy and carotic atherosclerosis in hypertension: a gender-based study. Blood Press 2013;22(3):138-43. https://doi.org/10.3109/08037051.2012.744151.

[15] Zanoli L, Lentini P, Boutouyrie P, Fatuzzo P, Granata A, Corrao S, et al. Pulse wave velocity differs between ulcerative colitis and chronic kidney disease. Eur J Intern Med 2018;47:36-42. 
[16] Van Bortel LM, Laurent S, Boutouyrie P, Chowienczyk P, Cruickshank JK, De Backer T, et al. European society of hypertension working group on vascular structure and function; european network for noninvasive investigation of large arteries. Expert consensus document on the measurement of aortic stiffness in daily practice using carotid-femoral pulse wave velocity. J Hypertens 2012;30:445-8.

[17] Chen CH, Nevo E, Fetics B, Pak PH, Yin FC, Maughan WL, et al. Estimation of central aortic pressure waveform by mathematical transformation of radial tonometry pressure. Validation of generalized transfer function. Circulation 1997;95:1827-36.

[18] Levey AS, Stevens LA, Schmid CH, Zhang YL, Castro 3rd AF, Feldman HI, et al., CKD-EPI (Chronic Kidney Disease Epidemiology Collaboration). A new equation to estimate glomerular filtration rate. Ann Intern Med 2009;150(9):604-12.

[19] Zanoli L, Rastelli S, Inserra G, Castellino P. Arterial structure and function in inflammatory bowel disease. World J Gastroenterol 2015;21(40):11304-11. https://doi.org/10.3748/wjg.v21.i40.11304.

[20] Navarro J, Redon J, Cea-Calvo L, Lozano JV, Fernandez-Perez C, Bonet A, et al. On behalf of the group of investigators of the ERICHTA Study. Metabolic syndrome, organ damage and cardiovascular disease in hypertension. The ERIC-HTA study. Blood Pres 2007; 16:20-7.

[21] Granata A, Clementi A, Virzì GM, Brocca A, de Cal M, Scarfia VR, et al. Cardiorenal syndrome type 4: from chronic kidney disease to cardiovascular impairment. Eur J Intern Med 2016;30:1-6.

[22] Briet M, Bozec E, Laurent S, Fassot C, London GM, Jacquot C, et al. Arterial stiffness and enlargement in mild-to-moderate chronic kidney disease. Kidney Int 2006;69(2):350-7.
[23] Kelly RP, Ting CT, Yang TM, Liu CP, Maughan WL, Chang MS, et al. Effective arterial elastance as index of arterial vascular load in humans. Circulation 1992;86:513-21.

[24] Mérillon JP, Ennezat PV, Guiomard A, Masquet-Gourgon C, Aumont MC, Gourgon R. Left ventricular performance is closely related to the physical properties of the arterial system: landmark clinical investigations in the 1970s and 1980s. Arch Cardiovasc Dis 2014;107(10):554-62.

[25] Spoto B, Pisano A, Zoccali C. Insulin resistance in chronic kidney disease: a systematic review. Am J Physiol Renal Physiol 2016; 311(6):F1087-108.

[26] Fang L, Ellims AH, Beale AL, Taylor AJ, Murphy A, Dart AM. Systemic inflammation is associated with myocardial fibrosis, diastolic dysfunction, and cardiac hypertrophy in patients with hypertrophic cardiomyopathy. Am J Transl Res 2017;9(11):5063-73.

[27] Giallauria F, Ling SM, Schreiber C, Maggio M, Shetty V, Muller D, et al. Arterial stiffness and bone demineralization: the Baltimore longitudinal study of aging. Am J Hypertens 2011;24(9):970-5.

[28] Reference Values for Arterial Stiffness' Collaboration. Determinants of pulse wave velocity in healthy people and in the presence of cardiovascular risk factors: 'establishing normal and reference values. Eur Heart J 2010;31(19):2338-50.

[29] Li X, Liu Y, Zheng Y, Wang P, Zhang Y. The effect of vitamin D supplementation on glycemic control in type 2 diabetes patients: a systematic review and meta-analysis. Nutrients 2018;10(3).

[30] Giallauria F, Milaneschi Y, Tanaka T, Maggio M, Canepa M, Elango P, et al. Arterial stiffness and vitamin D levels: the Baltimore longitudinal study of aging. J Clin Endocrinol Metab 2012; 97(10):3717-23. 